\title{
Creatinine-Based Formulae Poorly Match in the Classification of Hypofiltration or Hyperfiltration in a General Population of Adolescents: A Retrospective Analysis of a Cross-Sectional Study
}

\author{
Katarína Šebeková ${ }^{1 *}$, Radana Gurecká ${ }^{1,2}$, Lubomíra Tóthová ${ }^{1}$ and Ĺudmila Podracká ${ }^{3,4}$ \\ ${ }^{1}$ Faculty of Medicine, Institute of Molecular BioMedicine, Comenius University, Bratislava, Slovakia, ${ }^{2}$ Faculty of Medicine, \\ Institute of Medical Physics, Biophysics, Informatics and Telemedicine, Comenius University, Bratislava, Slovakia, \\ ${ }^{3}$ Department of Pediatrics, Faculty of Medicine, Comenius University, Bratislava, Slovakia, ${ }^{4}$ Department of Pediatrics, The \\ National Institute of Children's Health, Bratislava, Slovakia
}

OPEN ACCESS

Edited by:

Agnieszka Swiatecka-Urban, University of Virginia, United States

Reviewed by:

Mara Medeiros,

Federico Gómez Children's Hospital, Mexico

Emily Lauren Joyce,

Rainbow Babies and Children's Hospital, United States

*Correspondence:

Katarína Šebeková

kata.sebekova@gmail.com

Specialty section: This article was submitted to

Pediatric Nephrology,

a section of the journa

Frontiers in Pediatrics

Received: 03 June 2021 Accepted: 24 September 2021

Published: 28 October 2021

Citation:

Šebeková K, Gurecká R, Tóthová L and Podracká Ĺ (2021)

Creatinine-Based Formulae Poorly

Match in the Classification of

Hypofiltration or Hyperfiltration in a

General Population of Adolescents: A

Retrospective Analysis of a

Cross-Sectional Study.

Front. Pediatr. 9:719997.

doi: 10.3389/fped.2021.719997
Pediatric formulae to estimate glomerular filtration rate (eGFR) give a broad range of values. Their consistency in assigning the subjects as hypofiltrating or hyperfiltrating is unknown. In 1993 apparently healthy adolescents (53.4\% females) aged 14-17 years, we investigated the concordance of six creatinine-based formulae in the classification of the subjects into $\leq 5$ th or $\geq 95$ th percentile of eGFR, and the between-groups difference in the prevalence of cardiometabolic risk factors. Mean eGFR varied between 77 and $121 \mathrm{~mL} / \mathrm{min} / 1.73 \mathrm{~m}^{2}$. Arbitrary setting of hypofiltration or hyperfiltration to $5 \%$ returned 46 males and 53 females. At least one formula classified 89 males and 99 females as hypofiltrating and 105 males and 114 females as hyperfiltrating. All six formulae concordantly classified 15 males and 17 females as hypofiltrating and 9 and 14, respectively, as hyperfiltrating. Pairwise, formulae consistently classified hypofiltration in $42-87 \%$ of subjects with hyperfiltration in $28-94 \%$. According to two out of the six formulae, hyperfiltration was associated with an increased prevalence of obesity and obesity-associated comorbidities. Hypofiltrating subjects did not manifest chronic kidney disease-associated comorbidities. Further studies in different populations of healthy adolescents are needed before it is possible to conclude which creatinine-based formula is appropriate for the classification of hypofiltration and hyperfiltration in nonclinical cohorts.

Keywords: kidney function, children, creatinine, adolescents, cardiometabolic risk (factors), estimated glomerular filtration rate (eGFR)

\section{INTRODUCTION}

The worldwide increasing prevalence of hypertension and obesity in juveniles may predispose them to a rise in the manifestation of chronic kidney disease. Obesity-associated hyperfiltration is an antecedent of future chronic kidney disease $(1,2)$. Adolescent hypertension doubles the risk of end-stage renal disease regardless of the severity of hypertension and overweight 
(3). This urges the need for effective assessment of renal function in the general population of adolescents.

Serum creatinine-based estimated glomerular filtration rate (eGFR) is a widely used marker of renal function in both clinics and epidemiological studies. Despite that it is imperfect, it is generally employed because more accurate approaches for direct GFR assessment-renal and plasma clearance methods-can only be performed in specialized centers $(4,5)$. Urinary measurement of creatinine clearance requires the active cooperation of the subject in the accurate collection of urine over $24 \mathrm{~h}$. The more accurate cystatin C-based estimation of $\operatorname{GFR}(4,6,7)$ is several times more expensive than the creatininebased one. Thus, neither of these approaches is feasible in general practice or epidemiological studies. Formulae estimating GFR in adults, such as the MDRD or CKD-EPI equations $(8,9)$ do not apply to children and adolescents (10-12).

Derivation of universal pediatric equations faces several problems, such as sex differences in the growth spurt and muscle mass gain $(4,5,13,14)$. Currently, several equations derived from pediatric patients with renal disease or a general population of healthy subjects are available, but none of them ideally reflects measured GFR $(4,5)$. Studies in the general population of children and adolescents and pediatric patients with type 1 diabetes (T1D) comprehensively document the disparities and errors between measured and estimated GFR and show that different equations to estimate GFR return values in a broad range (13-16). However, it remains unclear whether different formulae consistently categorize adolescents into the lower and upper tail of eGFR distribution. Concordance in assigning is of clinical importance as a manifestation of low or high eGFR requires further diagnostic steps. To these points, we compared serum creatinine-based eGFR values obtained by six different equations in a large cohort of apparently healthy adolescents. We anticipated that the highest correlation across eGFR ranges as well as the highest consistency in assigning subjects into the tails of eGFR distribution, would be between pairs of equations derived from the same population. We also studied whether individuals with eGFR $\leq 5$ th percentile present morbidities that are commonly associated with decreased renal function and whether those displaying eGFR $\geq 95$ th percentile present obesity and obesity-associated risk markers.

\section{SUBJECTS AND METHODS}

This is a retrospective analysis of the data obtained in the crosssectional study "Respect for Health." The survey was launched in cooperation between the two local health authorities-the Department of Health of Bratislava Self-Governing Region and the Regional Public Health Authority of the Slovak Republic in Bratislava-and aimed to characterize the cardiometabolic health status of students attending public secondary schools in the Bratislava Region. Data were collected between November 2011 and December 2012. Acute illness, pregnancy, or lactation in females were exclusion criteria. Complete data on anthropometry and blood chemistry were obtained from 2960 students aged
12-23 years. For the current analysis, we extracted data on 14 17-year-old White Caucasian adolescents of Central European descent ( $n=1,993 ; 53.4 \%$ females).

The study was conducted according to the Declaration of Helsinki after the approval of the protocol by the Ethics Committee of the Bratislava Self-Governing Region. The decision to participate was voluntary. Signed informed consent was obtained from parents or legal guardians of participants.

\section{Measurements}

The study protocol has been explained in detail previously (17). Briefly, height, body weight, and blood pressure (BP) measurements were performed directly at high schools by trained staff, according to standard protocols (17). Body mass index (BMI) and waist-to-height ratio (WHtR) were calculated.

At appointed health centers, blood samples were collected after overnight fasting. Serum concentrations of glucose, highdensity lipoprotein cholesterol (HDL-C), triacylglycerols, highsensitivity C-reactive protein (CRP), insulin, and uric acid were analyzed in a central laboratory using standard analytical methods (ADVIA 2400 analyzer, Siemens, Erlangen, Germany). Serum creatinine was analyzed via a compensated, rate-blanked Jaffé reaction with an isotope dilution mass spectrometry (IDMS)-traceable calibrator (National Institute of Standards and technology, SRM 967). In spot urine, albumin (turbidimetrically) and creatinine concentrations were determined, and urinary albumin-to-creatinine ratio (ACR) was calculated.

\section{Estimation of Glomerular Filtration Rate}

Serum creatinine-derived eGFR was estimated using six formulae, i.e., the Schwartz-Lyon formula (18), the formula of Léger et al. (19), the revised Lund-Malmö (LM) formula (20), the Lund-Malmö formula with lean body mass extension (LMLBM) (21), and the full-age spectrum with Q-age (FAS-QA) or with Q-height extension (FAS-QH) (15), as follows:

Schwartz-Lyon formula (18)

$=k *($ height $[\mathrm{cm}] / \mathrm{sCrea}[\mu \mathrm{mol} / \mathrm{L}])$

$k=36.5$ in males aged $>13$ years.

$k=32.5$ in others.

\section{Léger's formula (19)}

$$
=56.7 * \text { weight }[\mathrm{kg}]+0.142 * h e i g h[\mathrm{~cm}]^{2} / \mathrm{sCrea}[\mu \mathrm{mol} / L]
$$

\section{LM formula (20)}

$$
=e^{X-0.0158 * \text { age }+0.438 * \ln (\text { age })}
$$

Males :

$\mathrm{X}=2.56+0.00968 *(180-\mathrm{sCrea}[\mu \mathrm{mol} / \mathrm{L}])$, if sCrea

$\leq 180 \mu \mathrm{mol} / \mathrm{L}$

$\mathrm{X}=2.56-0.926 * \ln (\mathrm{sCrea}[\mu \mathrm{mol} / \mathrm{L}] / 180)$, if sCrea

$\geq 180 \mu \mathrm{mol} / \mathrm{L}$

Females : 


$$
\begin{aligned}
& \mathrm{X}=2.50+0.0121 *(150-\mathrm{sCrea}[\mu \mathrm{mol} / \mathrm{L}]), \text { if sCrea } \\
& \leq 150 \mu \mathrm{mol} / \mathrm{L} \\
& \mathrm{X}=2.50-0.926 * \ln (\mathrm{sCrea}[\mu \mathrm{mol} / \mathrm{L}] / 150), \text { if sCrea } \\
& \geq 150 \mu \mathrm{mol} / \mathrm{L}
\end{aligned}
$$

\section{LM-LBM formula (20)}

$$
\begin{aligned}
& =e^{X-0.00587 * \text { age }+0.00977^{*} L B M} \\
& \mathrm{X}=4.95-0.0110 * \mathrm{sCrea}[\mu \mathrm{mol} / \mathrm{L}], \text { if } \mathrm{sCrea} \leq 150 \mu \mathrm{mol} / \mathrm{L} \\
& \mathrm{X}=8.58+0.0005 * \mathrm{sCrea}[\mu \mathrm{mol} / \mathrm{L}]-1.08 * \ln (\mathrm{sCrea}[\mu \mathrm{mol} / \mathrm{L}]) \\
& \text { if sCrea } \geq 150 \mu \mathrm{mol} / \mathrm{L}
\end{aligned}
$$

LBM:

$$
\begin{aligned}
\text { Males }: \mathrm{LBM}= & 1.10 * \text { weight }[\mathrm{kg}] \\
& -120 *(\text { weight }[\mathrm{kg}] / \text { height }[\mathrm{cm}])^{2} \\
\text { Females }: \mathrm{LBM}= & 1.07 * \text { weight }[\mathrm{kg}] \\
& -148 *(\text { weight }[\mathrm{kg}] / \text { height }[\mathrm{cm}])^{2}
\end{aligned}
$$

FAS-QH (15)

$$
\begin{aligned}
& =107.3 /(\text { sCrea }[m g / d L] / Q) \\
\mathrm{Q} & =3.94-13.4 *(\text { height }[\mathrm{m}])+17.6 *(\text { height }[\mathrm{m}])^{2} \\
& -9.84 *(\text { height }[\mathrm{m}])^{3}+2.04 *(\text { height }[\mathrm{m}])^{4}
\end{aligned}
$$

FAS-QA (15)

$$
\begin{aligned}
& =107.3 /(s C r e a[m g / d L] / Q) \\
\text { Males }: Q & =0.21+0.057 * \text { age }-0.0075 * \text { age }^{2}+0.00064 * \text { age }^{3} \\
& -0.000016^{*} \text { age }^{4} \\
\text { Females }: Q & =0.23+0.034 * \text { age }-0.0018 * \text { age }^{2}+0.00017 * \text { age }^{3} \\
& -0.0000051 * \text { age }^{4}
\end{aligned}
$$

eGFR is expressed in $\mathrm{mL} / \mathrm{min} / 1.73 \mathrm{~m}^{2}$, height is expressed in centimeters except for the FAS-QH formula, in which it is expressed in meters, sCrea: serum creatinine concentration is expressed in micromoles per liter $(\mu \mathrm{mol} / \mathrm{L})$ except for the FAS formulae, in which it is expressed in $\mathrm{mg} / \mathrm{dL}$ (conversion factor: 88.42), age is expressed in years, body weight in kilograms, Ln: natural logarithm, e: the base of the natural logarithm.

Arbitrarily, hypofiltration and hyperfiltration were set at the formula- and sex-specific $\leq 5$ th and $\geq 95$ th eGFR percentiles, respectively. We also used the conventional cutoffs: $\leq 75$ $\mathrm{mL} / \mathrm{min} / 1.73 \mathrm{~m}^{2}$ and $\geq 135 \mathrm{~mL} / \mathrm{min} / 1.73 \mathrm{~m}^{2}$ as recommended for adolescents $(22,23)$.

\section{Definition of Cardiometabolic Risk Factors}

The presence of general overweight and obesity was classified using the international age- and sex-specific cutoff points (24); that of central obesity is WHtR $\geq 0.5$ (25). The presence of elevated systolic BP (SBP $\geq 130 \mathrm{mmHg}$ ) or diastolic BP $(\mathrm{DBP} \geq 85 \mathrm{mmHg}$ ), elevated triacylglycerols (TAG $\geq$ $1.7 \mathrm{mmol} / \mathrm{L}$ ), low HDL-C (males: $<1.03 \mathrm{mmol} / \mathrm{L}$, females: $<1.29 \mathrm{mmol} / \mathrm{L}$ ), increased atherogenic index of plasma $[=\log$
(TAG/HDL-C) $] \geq 0.11$ (26), fasting glycemia $(\geq 5.6 \mathrm{mmol} / \mathrm{L})$, elevated uric acid levels ( $\geq 340 \mu \mathrm{mol} / \mathrm{L}$ in females, $\geq 420 \mu \mathrm{mol} / \mathrm{L}$ in males), the concentration of fasting insulin $\geq 20 \mu \mathrm{IU} / \mathrm{mL}$ (27), or CRP $>3 \mathrm{mg} / \mathrm{L}$ (28) were considered as markers of increased cardiometabolic risk. Microalbuminuria was classified as ACR $2.5-25.0 \mathrm{mg} / \mathrm{mmol}$ in males and $3.5-35.0 \mathrm{mg} / \mathrm{mmol}$ in females (29).

\section{Statistics}

Data characterizing the cohort are given as mean \pm SD for not normally distributed data as the median (interquartile range). Body weight, height, and BMI $z$-scores were calculated using the age- and sex-specific national reference data from 2001 (30). The descriptive characteristic of eGFR obtained by different formulae is given as the mean, SD, and 5th and 95th percentiles separately for males and females. The prevalence of subjects presenting eGFR under or above the conventional cutoffs for hypofiltration and hyperfiltration in adolescents is given as count and percentage. Between-sex differences in GFR estimated by different formulae were compared using the twosided unpaired Student's $t$-test. Mutual regressions of eGFR values obtained by six equations were expressed as coefficients of determination $\left(R^{2}\right)$. The agreement of different formulae in returning individuals' eGFR within the lower or upper tail of eGFR distribution, i.e., $\leq 5$ th or $\geq 95$ th percentile, was examined either as a match of designation by all six equations or pairwise. Fisher's exact test was used to compare the prevalence of cardiometabolic risk factors between subjects within the lower and the upper tail of each eGFR distribution. Data are presented as means or as counts. A $P<0.05$ was considered statistically significant. Analyses were performed by using the SPSS v.16 for Windows software (SPSS Inc., Chicago, IL, USA).

\section{RESULTS}

Cohort characteristics are given in Table 1. Males and females were of similar age and had mean serum creatinine concentrations of $74.8 \pm 13.2$ and $60.9 \pm 7.6 \mu \mathrm{mol} / \mathrm{L}$, respectively. Forty-three (4.5\%) males and 13 (1.2\%) females presented serum creatinine levels above their age-specific upper reference limit defined by Pottel et al. (23).

\section{Glomerular Filtration Rate Estimated via Different Formulae}

Descriptive characteristics of data on eGFR obtained by each formula are given in Table 2. In both sexes, the LM equation yielded the lowest mean eGFR values and the lowest cutoffs for the 5th and 95th percentiles. The highest values were obtained using Léger's formula. The difference between means returned by Léger's vs. LM formula reached about $41 \mathrm{~mL} / \mathrm{min} / 1.73 \mathrm{~m}^{2}$ in males and $45 \mathrm{~mL} / \mathrm{min} / 1.73 \mathrm{~m}^{2}$ in females. At the 5 th percentile, it corresponded to about $29 \mathrm{~mL} / \mathrm{min} / 1.73 \mathrm{~m}^{2}$ and $33 \mathrm{~mL} / \mathrm{min} / 1.73$ $\mathrm{m}^{2}$, respectively; at the $95 \mathrm{th}$, to about $56 \mathrm{~mL} / \mathrm{min} / 1.73 \mathrm{~m}^{2}$ in males and $62 \mathrm{~mL} / \mathrm{min} / 1.73 \mathrm{~m}^{2}$ in females.

Employing the LM equation, $43 \%$ of males presented eGFR $<$ $75 \mathrm{~mL} / \mathrm{min} / 1.73 \mathrm{~m}^{2}$; with the Schwartz-Lyon formula assigned to this category, about $11 \%$ of males; with the other four equations, 
the prevalence was $<3 \%$ (Table 2). In females, the prevalence of eGFR $<75 \mathrm{~mL} / \mathrm{min} / 1.73 \mathrm{~m}^{2}$ reached about 47,8 , and $0-$ $1 \%$, respectively. Using Léger's and the FAS-QH formulae, the prevalence of eGFR $\geq 135 \mathrm{~mL} / \mathrm{min} / 1.73 \mathrm{~m}^{2}$ reached about 19 and $15 \%$, respectively, in males and 22 and $6 \%$ in females. The

TABLE 1 | Characteristics of the studied population.

\begin{tabular}{lcc}
\hline & Males & Females \\
\hline$n$ & 929 & 1,046 \\
Age, years & $16.1 \pm 0.8$ & $16.2 \pm 0.8$ \\
Height, $\mathrm{cm}$ & $178.8 \pm 7.0$ & $165.7 \pm 6.2$ \\
Height $Z$-score & $0.15 \pm 1.02$ & $0.01 \pm 1.00$ \\
Body weight, kg & $73.1 \pm 14.2$ & $59.9 \pm 10.2$ \\
Body weight Z-score & $0.62 \pm 1.30$ & $0.32 \pm 1.13$ \\
BMl, $\mathrm{kg} / \mathrm{m}^{2}$ & $22.8 \pm 3.9$ & $21.8 \pm 3.4$ \\
BMl Z-score & $0.61 \pm 1.28$ & $0.34 \pm 1.10$ \\
WHtR & $0.44 \pm 0.05$ & $0.43 \pm 0.05$ \\
SBP, $\mathrm{mm} \mathrm{Hg}$ & $122 \pm 12$ & $107 \pm 9$ \\
FPG, $\mathrm{mmol} / \mathrm{L}$ & $4.9 \pm 0.5$ & $4.7 \pm 0.8$ \\
FPI, $\mu \mathrm{UI} / \mathrm{mL}$ & $9.4(7.9 ; 13.0)$ & $9.9(7.4 ; 13.4)$ \\
HDL-C, mmol/L & $1.25 \pm 0.23$ & $1.48 \pm 0.28$ \\
TAG, $\mathrm{mmol} / \mathrm{L}$ & $0.85 \pm 0.43$ & $0.85 \pm 0.37$ \\
AlP & $-0.20 \pm 0.22$ & $-0.27 \pm 0.20$ \\
Uric acid, $\mu \mathrm{mol} / \mathrm{L}$ & $355 \pm 60$ & $262 \pm 52$ \\
ACR, $\mathrm{mg} / \mathrm{mmol}$ & $0.4(0.2 ; 0.6)$ & $0.5(0.3 ; 0.8)$ \\
$\mathrm{CRP}, \mathrm{mg} / \mathrm{L}$ & $0.4(0.2 ; 1.0)$ & $0.4(0.2 ; 1.0)$ \\
& &
\end{tabular}

$B M I$, body mass index; WHtR, waist-to-height ratio; SBP, systolic blood pressure; FPG, fasting plasma glucose; FPI, fasting plasma insulin; HDL-C, high-density lipoprotein cholesterol; TAG, triacylglycerols; AIP, atherogenic index of plasma; ACR, urinary albuminto-creatinine ratio; CRP, C-reactive protein, data given as mean \pm standard deviation or as median (interquartile range), Z-scores were calculated based on the national sex- and age-specific standards. remaining four formulae returned prevalence between 0.1 and $2 \%$ in both sexes.

Mean eGFR calculated using the Schwartz-Lyon formula did not differ between the sexes. Léger's, LM-LBM, and FAS-QA equations gave higher mean eGFR in females compared with males; the opposite was observed using the LM and FAS-QH equations (Table 2 ).

For a better understanding of the differences, chart flows of serum creatinine concentration and eGFR values returned by six formulae across the 1st to 99th percentile were plotted (Figures 1A,B). Among two formulae derived from different cohorts of children with chronic kidney disease in both sexes, Léger's equation returned higher eGFR values compared with the Schwartz-Lyon equation. As for formulae derived from the same populations, in both sexes, the LM-LBM equation returned higher eGFR values compared with the LM formula. From eGFR $>82 \mathrm{~mL} / \mathrm{min} / 1.73 \mathrm{~m}^{2}$ in males and about $104 \mathrm{~mL} / \mathrm{min} / 1.73 \mathrm{~m}^{2}$ in females, FAS-QH gave higher eGFR values compared with FAS-QA, particularly in males (Figures 1A,B).

In males, LM-LBM, FAS-QA, and FAS-QH equations returned similar eGFR values at the 1 st percentile (Figure 1A). Thereafter, the slope of the LM-LBM equation flattened, and from the 95th percentile onward (about $113 \mathrm{~mL} / \mathrm{min} / 1.73 \mathrm{~m}^{2}$ ), LM-LBM eGFR values copied those returned by the Schwartz-Lyon equation. Up to the 5th percentile, both FAS formulae gave almost identical eGFR values. Although the slope of the FAS-QA equation showed minor variations across the percentiles, that of the FAS-QH equation rose more steeply, matching the values returned by Léger's formula at the 95th and 99th percentiles (i.e., $\geq 155$ $\mathrm{mL} / \mathrm{min} / 1.73 \mathrm{~m}^{2}$ ).

In females, eGFR lines of both FAS and the LM-LBM formulae started to deviate above the 50th percentile, corresponding to an eGFR of $102-106 \mathrm{~mL} / \mathrm{min} / 1.73 \mathrm{~m}^{2}$. Thereafter, the slope of the LM-LBM formula flattened, reaching the 99th percentile value

TABLE 2 | Descriptive characteristics of estimated glomerular filtration rate returned by six creatinine-based formulae in males and females.

\begin{tabular}{|c|c|c|c|c|c|c|}
\hline eGFR formula & Sch-L & Léger & LM & LM-LBM & FAS-QH & FAS-QA \\
\hline \multicolumn{7}{|l|}{ Males } \\
\hline Mean & 89.2 & 118.6 & 78.0 & 95.4 & 112.5 & 101.1 \\
\hline Standard deviation & 12.9 & 20.8 & 11.8 & 12.0 & 22.0 & 14.1 \\
\hline 5th percentile & 71.1 & 90.6 & 61.5 & 78.0 & 83.2 & 81.5 \\
\hline 95th percentile & 111.7 & 155.1 & 98.8 & 114.1 & 153.6 & 125.8 \\
\hline$\leq 75 \mathrm{~mL} / \mathrm{min} / 1.73 \mathrm{~m}^{2}, n(\%)$ & $98(10.5)$ & $5(0.5)$ & $398(42.8)$ & $26(2.8)$ & $16(1.7)$ & $11(1.2)$ \\
\hline$\geq 135 \mathrm{~mL} / \mathrm{min} / 1.73 \mathrm{~m}^{2}, n(\%)$ & $4(0.4)$ & $175(18.8)$ & $1(0.1)$ & $1(0.1)$ & $135(14.5)$ & $14(1.5)$ \\
\hline \multicolumn{7}{|l|}{ Females } \\
\hline Mean & 89.8 & 121.6 & 76.5 & 101.8 & 107.5 & 105.2 \\
\hline Standard deviation & 11.3 & 18.6 & 10.0 & 9.1 & 16.0 & 13.4 \\
\hline$P$ (vs. males) & 0.276 & 0.001 & 0.002 & $<0.001$ & $<0.001$ & $<0.001$ \\
\hline 5th percentile & 72.7 & 94.7 & 61.8 & 86.9 & 84.5 & 85.4 \\
\hline 95th percentile & 108.9 & 155.3 & 93.4 & 116.8 & 137.9 & 128.7 \\
\hline$\leq 75 \mathrm{~mL} / \mathrm{min} / 1.73 \mathrm{~m}^{2}, n(\%)$ & $80(7.5)$ & 0 & $499(46.9)$ & $3(0.3)$ & $6(0.6)$ & $4(0.4)$ \\
\hline$\geq 135 \mathrm{~mL} / \mathrm{min} / 1.73 \mathrm{~m}^{2}, n(\%)$ & $1(0.1)$ & $229(21.5)$ & $1(0.1)$ & $1(0.1)$ & 64 (6.3) & $21(2.1)$ \\
\hline
\end{tabular}

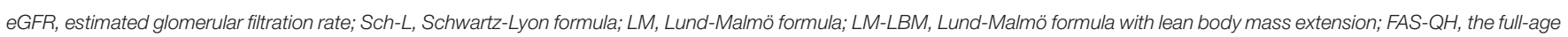
spectrum formula with $Q$-height extension; FAS-QA, the full-age spectrum formula with $Q$-age extension, significant $p$ is given in bold. 


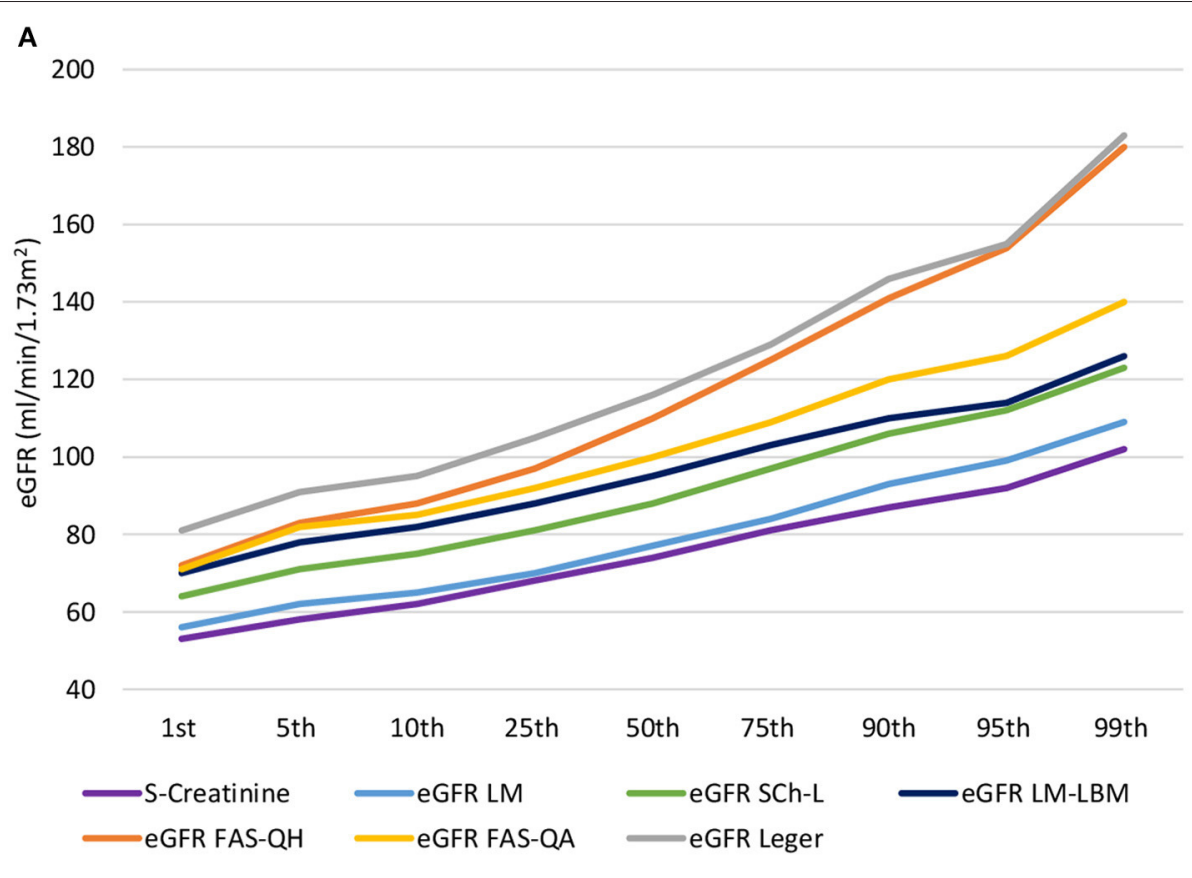

B

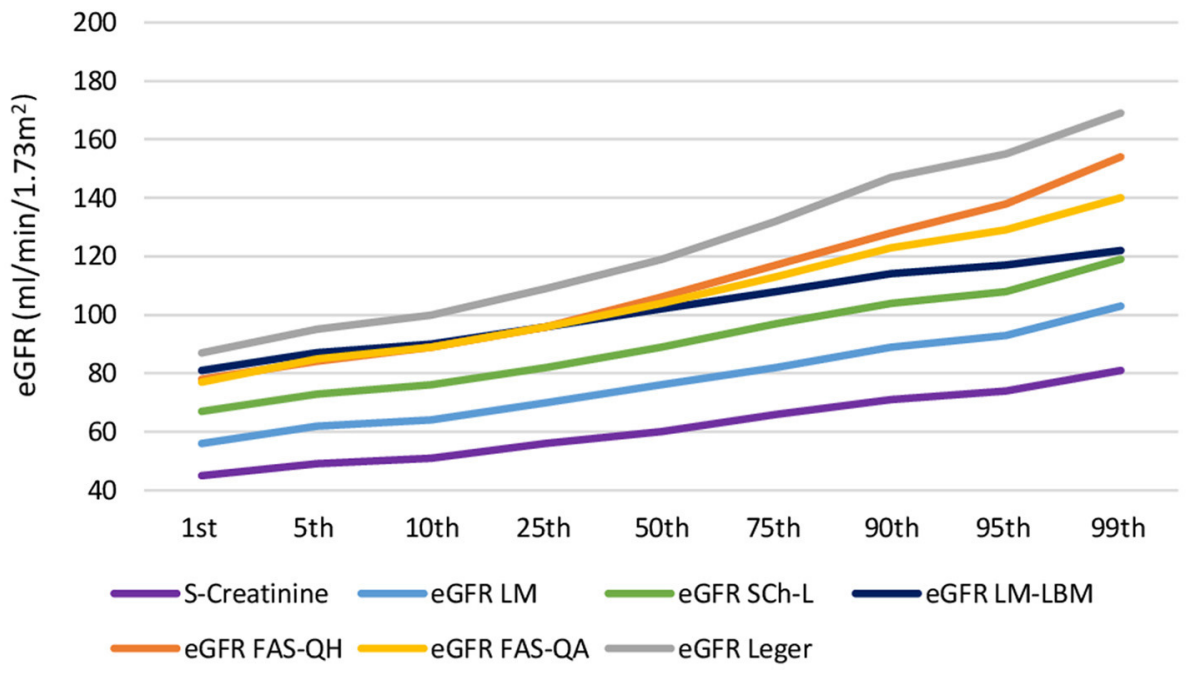

FIGURE 1 | Chart flow of serum creatinine concentration and estimated glomerular filtration rate values returned by six formulae across the 1st to 99th percentile in (A) males and (B) females. eGFR, estimated glomerular filtration rate; Sch-L, Schwartz-Lyon formula; LM, Lund-Malmö formula; LM-LBM, Lund-Malmö formula with lean body mass extension; FAS-QH, the full-age spectrum formula with Q-height extension; FAS-QA the full-age spectrum formula with $Q$-age extension.

similar to that returned by the Schwartz-Lyon equation, and that of the FAS-QH equation rose more rapidly than that of FAS$\mathrm{QA}$, but in contrast to males, it did not reach values returned by Léger's equation at the 99th percentile (Figure 1B).

In both sexes, coefficients of determination between LM and Schwartz-Lyon, LM and FAS-QA, and LM-LBM and Léger's equations were $>0.9$ (Table 3 ). Schwartz-Lyon showed a good correlation with LM-LBM or FAS-QA formulae both in males and females, and in females, it reached a value $>0.8$ also vs. the FAS-QH formula. In both sexes, equations derived from the same population, i.e., FAS-QH vs. FAS-QA or LM vs. LM-LBM, showed poor association (Table 3).

\section{Consistency of Six Formulae in the Classification of Subjects as Hypofiltrating or Hyperfiltrating}

Arbitrary setting of the prevalence to 5\% returned 46 males and 53 females. 
TABLE 3 | Coefficients of determination between estimated glomerular filtration rate returned by six formulae in males (right upper corner) and females (lower left corner).

\begin{tabular}{|c|c|c|c|c|c|c|}
\hline & Sch-L & Léger & LM & LM-LBM & FAS-QH & FAS-QA \\
\hline Sch-L & - & 0.691 & 0.925 & 0.846 & 0.637 & 0.841 \\
\hline Léger & 0.712 & - & 0.534 & 0.912 & 0.679 & 0.507 \\
\hline LM & 0.910 & 0.546 & - & 0.736 & 0.377 & 0.916 \\
\hline LM-LBM & 0.830 & 0.929 & 0.709 & - & 0.643 & 0.674 \\
\hline FAS-QH & 0.815 & 0.731 & 0.551 & 0.736 & - & 0.507 \\
\hline FAS-QA & 0.872 & 0.523 & 0.976 & 0.669 & 0.523 & - \\
\hline
\end{tabular}

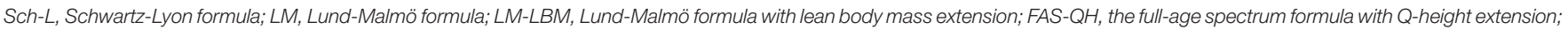
FAS-QA, the full-age spectrum formula with Q-age extension.

TABLE 4 | Consistency between pairs of equations in assigning males and females into the $\leq 5$ th percentile (the upper right corner) and into the $\geq 95$ th percentile (the lower left corner) of each eGFR distribution.

\begin{tabular}{|c|c|c|c|c|c|c|}
\hline & Sch-L & Léger & LM & LM-LBM & FAS-QH & FAS-QA \\
\hline \multicolumn{7}{|l|}{ Males } \\
\hline Sch-L & - & $61 \%$ & $78 \%$ & $80 \%$ & $63 \%$ & $67 \%$ \\
\hline Léger & $48 \%$ & - & $48 \%$ & $72 \%$ & $72 \%$ & $43 \%$ \\
\hline LM & $83 \%$ & $39 \%$ & - & $70 \%$ & $46 \%$ & $80 \%$ \\
\hline LM-LBM & $59 \%$ & $85 \%$ & $48 \%$ & - & $67 \%$ & $65 \%$ \\
\hline FAS-QH & $46 \%$ & $48 \%$ & $30 \%$ & $52 \%$ & - & $43 \%$ \\
\hline FAS-QA & $67 \%$ & $37 \%$ & $76 \%$ & $44 \%$ & $28 \%$ & - \\
\hline \multicolumn{7}{|l|}{ Females } \\
\hline Sch-L & - & $62 \%$ & $83 \%$ & $74 \%$ & $71 \%$ & $69 \%$ \\
\hline Léger & $57 \%$ & - & $49 \%$ & $83 \%$ & $67 \%$ & $42 \%$ \\
\hline LM & $68 \%$ & $40 \%$ & - & $62 \%$ & $54 \%$ & $87 \%$ \\
\hline LM-LBM & $57 \%$ & $94 \%$ & $40 \%$ & - & $67 \%$ & $53 \%$ \\
\hline FAS-QH & $70 \%$ & $53 \%$ & $45 \%$ & $55 \%$ & - & $42 \%$ \\
\hline FAS-QA & $70 \%$ & $42 \%$ & $79 \%$ & $42 \%$ & $47 \%$ & - \\
\hline
\end{tabular}

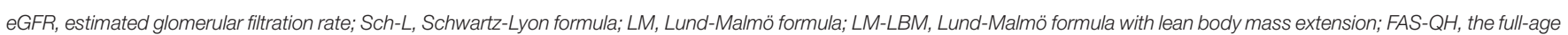
spectrum formula with Q-height extension; FAS-QA, the full-age spectrum formula with Q-age extension.

At least one formula classified 89 males as hypofiltrating. Of those, six formulae matched in the classification of 15 males $(17 \%$ out of 89$) ; 10$ (11\%) subjects were concordantly classified by any five as well as by any four formulae out of six; three equations consistently identified 12 males (13\%), two formulae achieved concordance in 19 males (21\%), and in 23 males (26\%) only one out of six formulae indicated hypofiltration.

As for the concordance of classification between the pairs of equations, the Schwartz-Lyon vs. LM-LBM and LM vs. FASQA showed the highest ( $80 \%$, both), and two FAS equations the lowest $(43 \%)$ concordance in sorting males to the lower tail of eGFR distribution (Table 4). For example, of 46 males classified as hypofiltrating by the Schwartz-Lyon formula, the LM-LBM equation classified as hypofiltrating 37 , and $43 \%$ concordance observed for FAS formulae means that the second formula denotes as hypofiltrating 20 out of 46 subjects in the lower tail of the distribution of the first formula.

Among females, 99 were classified as presenting hypofiltration by at least one formula. Of them, 17 were concordantly classified as hypofiltrating by all six formulae, any five or any four formulae matched in the classification of 12 females, three formulae concordantly classified 11, and any two equations reached concordance in 26 individuals. Twenty-one females were classified as hypofiltrating by any one of the six formulae. This corresponded to $17,12,12,11,26$, and $21 \%$ of 99 subjects.

In females, FAS-QA vs. LM, LM-LBM vs. Léger's, and LM vs. Schwartz-Lyon equations yielded the highest (87-83\%) matches in the classification of hypofiltration, and the poorest concordance (42\%) was between the FAS formulae (Table 4). For example, FAS-QA and LM formulae indicating $87 \%$ matching concordantly assigned as hypofiltrating 46 of 53 females, in the case of the two FAS formulae, it was 22 out of 53.

One hundred five males were classified as hyperfiltrating by at least one formula. Six formulae matched in the classification of nine males (about 9\% out of 105); eight (8\%) subjects were concordantly classified by any five, 12 (11\%) by any four formulae out of six; three equations consistently identified 18 males (17\%), two formulae achieved concordance in 22 males (21\%), and in 36 males (34\%), only one out of six formulae indicated hyperfiltration. Léger's and LM-LBM, and SchL and LM formulae reached a consistency of 85 and $83 \%$, respectively, in assigning males as hyperfiltrating (Table 4). 
TABLE 5 | Mean estimated glomerular filtration rate, mean age, and mean height of males and females assigned by six formulae to the lower ( $\leq 5$ th) and the upper ( $\geq 95$ th) tail of each distribution.

\begin{tabular}{|c|c|c|c|c|c|c|c|c|c|}
\hline \multirow[t]{2}{*}{ Formula } & \multicolumn{3}{|c|}{ eGFR, mL/min/1.73 $\mathrm{m}^{2}$} & \multicolumn{3}{|c|}{ Age, years } & \multicolumn{3}{|c|}{ Height, cm } \\
\hline & $\leq 5$ th & $\geq 95$ th & $p$ & $\leq 5$ th & $\geq 95$ th & p & $\leq 5$ th & $\geq 95$ th & $p$ \\
\hline \multicolumn{10}{|l|}{ Males } \\
\hline Sch-L & 65 & 120 & $<0.001$ & 16.4 & 15.5 & $<0.001$ & 176.3 & 178.3 & 0.215 \\
\hline Léger & 82 & 172 & $<0.001$ & 16.3 & 15.8 & 0.013 & 173.1 & 183.3 & $<0.001$ \\
\hline LM-LBM & 70 & 121 & $<0.001$ & 16.4 & 16.7 & $<0.001$ & 176.4 & 182.7 & $<0.001$ \\
\hline FAS-QH & 75 & 168 & $<0.001$ & 16.3 & 15.9 & 0.055 & 171.2 & 189.1 & $<0.001$ \\
\hline FAS-QA & 74 & 134 & $<0.001$ & 16.0 & 16.0 & 1.000 & 181.3 & 175.7 & 0.001 \\
\hline \multicolumn{10}{|l|}{ Females } \\
\hline Sch-L & 82 & 133 & $<0.001$ & 16.4 & 16.2 & 0.101 & 164.5 & 168.8 & $<0.001$ \\
\hline FAS-QA & 81 & 136 & $<0.001$ & 15.9 & 16.4 & 0.002 & 168.0 & 164.5 & 0.003 \\
\hline
\end{tabular}

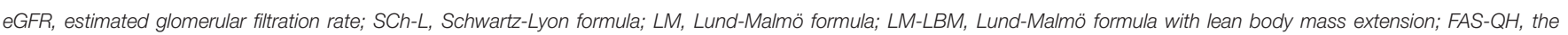
full-age spectrum formula with $Q$-height extension; FAS-QA, the full-age spectrum formula with $Q$-age extension, significant $p$ is given in bold.

The poorest concordance (28\%) was revealed between the two FAS formulae.

Among females, 114 were classified as presenting hyperfiltration by at least one formula. All six, and any five, four, three, and two formulae concordantly classified 14, 10, 12, 13, and 33 females, respectively; corresponding to $12,9,11,11$, and $29 \%$ out of 114 subjects. One formula indicated hyperfiltration in 32 females $(28 \%)$. As in males, Léger's and LM-LBM formulae showed the highest matching (94\%) in the classification of hyperfiltration, followed by LM with FAS-QA (79\%) (Table 4). The poorest agreement (40\%) was observed between LM and LBM as well as between LM and Léger's equations.

\section{Comparison of Mean Age, Height, and the Prevalence of Cardiometabolic Risk Markers Among Adolescents With eGFR $\leq 5$ th vs. $\geq 95$ th Percentile by Various Pediatric GFR Estimating Equations}

Using all but FAS equations, males classified as hypofiltrating were significantly younger (by 4-11 months) compared with their peers assigned to the upper tail of eGFR distribution (Table 5). With the height-independent equations, males presenting in the eGFR $\leq 5$ th percentile were taller. The opposite was observed employing the height-dependent formulae; with the FAS-QH equation, the means differed by almost $18 \mathrm{~cm}$ (Table 5).

Using the Léger's and LM-LBM equations, the prevalence of central obesity, general overweight/obesity, elevated BP, low HDL-C, elevated atherogenic index, and CRP $>3 \mathrm{mg} / \mathrm{L}$ was significantly higher in males at the upper tail of eGFR distribution vs. the lower tail (Table 6). The prevalence of general obesity was also higher by Schwartz-Lyon and FAS-QA formulae. All but FAS-QA equations indicated a higher prevalence of elevated fasting insulin in subjects with eGFR $\geq 95$ th percentile. The prevalence of elevated triacylglycerols, uric acid, and that of microalbuminuria did not differ between the groups (Table 6).

With the LM-LBM formula, females at the upper tail of eGFR distribution were about 5 months younger than their peers at the lower tail, and the FAS-QA equation returned the opposite (Table 5). As in males, LM and FAS-QA equations indicated that females at the upper tail of distribution were shorter compared with their hypofiltrating peers, and the opposite was observed employing the height-dependent formulae. With the FAS-QH equation, the height difference reached about $14 \mathrm{~cm}$.

Using the FAS-QA formula, females manifesting eGFR $\leq 5$ th percentile more likely suffered from hyperuricemia than their counterparts with eGFR $\geq 95$ th percentile (Table 7 ). Léger's and LM-LBM equations indicated a higher prevalence of central obesity, general overweight/obesity or obesity, elevated fasting insulin, and CRP levels and low HDL-C in females at the upper tails of eGFR distributions compared with those on the lower ones. None of the equations indicated a difference in the prevalence of elevated BP, hypertriacylglycerolemia, elevated atherogenic index, or microalbuminuria.

\section{DISCUSSION}

We explored the agreement between six creatinine-based formulae for estimation of GFR in a cohort of apparently healthy adolescents, intending to find out which equations match the best in the classification of hyperfiltration and hypofiltration. As expected, different formulae returned a broad range of eGFR values. Importantly, there was not a simple numerical shift 
TABLE 6 | The prevalence of cardiometabolic risk factors in males with estimated glomerular filtration rate $\leq 5$ th vs. that of $\geq 95$ th according to six creatinine-based equations.

\begin{tabular}{|c|c|c|c|c|c|c|c|c|c|c|c|c|c|c|c|c|c|c|}
\hline \multirow[t]{3}{*}{ Formula } & \multicolumn{3}{|c|}{ WHtR $\geq 0.5$} & \multicolumn{3}{|c|}{ BMI Ow/Ob OITF } & \multicolumn{3}{|c|}{ BMI Ob IOTF } & \multicolumn{3}{|c|}{$\mathrm{SBP} \geq 130$ or $\mathrm{DBP} \geq 85 \mathrm{~mm} \mathrm{Hg}$} & \multicolumn{3}{|c|}{ Glycemia $\geq 5.6 \mathrm{mmol} / \mathrm{L}$} & \multicolumn{3}{|c|}{ Insulin $\geq 20 \mu \mathrm{UI} / \mathrm{mL}$} \\
\hline & \multicolumn{2}{|c|}{ eGFR perc. } & \multirow{2}{*}{$p$} & \multicolumn{2}{|c|}{ eGFR perc. } & \multirow{2}{*}{$p$} & \multicolumn{2}{|c|}{ eGFR perc. } & \multirow[t]{2}{*}{$p$} & \multicolumn{2}{|c|}{ eGFR perc. } & \multirow{2}{*}{$p$} & \multicolumn{2}{|c|}{ eGFR perc. } & \multirow{2}{*}{$p$} & \multicolumn{2}{|c|}{ eGFR perc. } & \multirow{2}{*}{$p$} \\
\hline & $\leq 5$ th & $\geq 95$ th & & $\leq 5$ th & $\geq 95$ th & & $\leq 5$ th & $\geq 95$ th & & $\leq 5$ th & $\geq 95$ th & & $\leq 5$ th & $\geq 95$ th & & $\leq 5$ th & $\geq 95$ th & \\
\hline Sch-L & 4 & 10 & 0.145 & 15 & 18 & 0.664 & 1 & 8 & 0.030 & 10 & 9 & 1.000 & 2 & 2 & 1.000 & 2 & 11 & 0.014 \\
\hline Léger & 1 & 29 & $<0.001$ & 6 & 39 & $<0.001$ & 0 & 25 & $<0.001$ & 5 & 19 & 0.002 & 3 & 2 & 1.000 & 3 & 21 & $<0.001$ \\
\hline LM & 4 & 10 & 0.089 & 15 & 16 & 0.828 & 2 & 8 & 0.050 & 12 & 7 & 0.304 & 3 & 1 & 0.617 & 3 & 10 & 0.040 \\
\hline LM-LBM & 3 & 22 & $<0.001$ & 10 & 35 & $<0.001$ & 1 & 18 & $<0.001$ & 9 & 17 & 0.104 & 3 & 2 & 1.000 & 3 & 17 & 0.001 \\
\hline FAS-QH & 4 & 8 & 0.345 & 12 & 18 & 0.266 & 1 & 7 & 0.059 & 9 & 20 & 0.024 & 3 & 2 & 1.000 & 1 & 11 & 0.004 \\
\hline FAS-QA & 3 & 10 & 0.069 & 13 & 14 & 1.000 & 1 & 8 & 0.030 & 11 & 10 & 1.000 & 2 & 2 & 1.000 & 3 & 10 & 0.069 \\
\hline \multirow[t]{3}{*}{ Formula } & \multicolumn{3}{|c|}{$\mathrm{HDL}-\mathrm{C}<1.29 \mathrm{mmol} / \mathrm{L}$} & \multicolumn{3}{|c|}{$\mathrm{TAG} \geq 1.7 \mathrm{mmol} / \mathrm{L}$} & \multicolumn{3}{|c|}{ AIP $\geq 0.11$} & \multicolumn{3}{|c|}{$\mathrm{UA} \geq 340 \mu \mathrm{mol} / \mathrm{L}$} & \multicolumn{3}{|c|}{$\mathrm{ACR} \geq 3.5 \mathrm{mg} / \mathrm{mmol}$} & \multicolumn{3}{|c|}{$\mathrm{CRP}>3 \mathrm{mg} / \mathrm{L}$} \\
\hline & \multicolumn{2}{|c|}{ eGFR perc } & \multirow[t]{2}{*}{$p$} & \multicolumn{2}{|c|}{ eGFR perc } & \multirow[t]{2}{*}{$p$} & \multicolumn{2}{|c|}{ eGFR perc } & $p$ & eGF & perc & $p$ & eGF & perc & $p$ & eGF & perc & $p$ \\
\hline & $\leq 5$ th & $\geq 95$ th & & $\leq 5$ th & $\geq 95$ th & & $\leq 5$ th & $\geq 95$ th & & $\leq 5$ th & $\geq 95$ th & & $\leq 5$ th & $\geq 95$ th & & $\leq 5$ th & $\geq 95$ th & \\
\hline Sch-L & 8 & 8 & 1.000 & 0 & 3 & 0.242 & 2 & 5 & 0.434 & 7 & 3 & 0.315 & 2 & 2 & 1.000 & 2 & 8 & 0.090 \\
\hline Léger & 8 & 19 & 0.021 & 1 & 6 & 0.111 & 1 & 11 & 0.004 & 5 & 11 & 0.168 & 1 & 0 & 1.000 & 1 & 9 & 0.015 \\
\hline LM & 9 & 8 & 1.000 & 2 & 3 & 0.677 & 3 & 5 & 0.486 & 8 & 2 & 0.091 & 2 & 3 & 0.677 & 1 & 6 & 0.059 \\
\hline LM-LBM & 9 & 16 & 0.159 & 2 & 6 & 0.267 & 2 & 10 & 0.027 & 6 & 9 & 0.574 & 2 & 0 & 0.495 & 1 & 8 & 0.030 \\
\hline FAS-QH & 8 & 10 & 0.793 & 1 & 2 & 1.000 & 2 & 4 & 0.677 & 9 & 4 & 0.231 & 1 & 2 & 1.000 & 2 & 6 & 0.267 \\
\hline FAS-QA & 10 & 8 & 0.793 & 2 & 3 & 1.000 & 2 & 5 & 0.434 & 10 & 3 & 0.069 & 1 & 2 & 1.000 & 2 & 8 & 0.090 \\
\hline
\end{tabular}

The prevalence is given as the number of males presenting the risk factor out of 46/group.

Formulae: Sch-L, Schwartz-Lyon; LM, Lund-Malmo; LM-LMB, Lund-Malmo with lean body mass; FAS-QH, Full-age Spectrum with Q-height; FAS-QA, Full-age Spectrum with Q-age.

WHtR, waist-to-height ratio; BMI, body mass index; ow/ob, overweight/obese; OITF, The International Obesity Taskforce; SBP, systolic blood pressure; DBP, diastolic blood pressure; HDL-C, high-density lipoprotein cholesterol; eGFR, estimated glomerular filtration rate; TAG, triacylglycerols; AIP, atherogenic index of plasma; ACR, UA, uric acid; urinary albumin-to-creatinine ratio; CRP, C-reactive protein, significant p is given in bold. 
TABLE 7 | The prevalence of cardiometabolic risk factors in females with estimated glomerular filtration rate $\leq 5$ th vs. that of $\geq 95$ th according to six creatinine-based equations.

\begin{tabular}{|c|c|c|c|c|c|c|c|c|c|c|c|c|c|c|c|c|c|c|}
\hline \multirow[t]{3}{*}{ Formula } & \multicolumn{3}{|c|}{ WHtR $\geq 0.5$} & \multicolumn{3}{|c|}{ BMI Ow/Ob OITF } & \multicolumn{3}{|c|}{ BMI Ob IOTF } & \multicolumn{3}{|c|}{$\mathrm{SBP} \geq 130$ or $\mathrm{DBP} \geq 85 \mathrm{~mm} \mathrm{Hg}$} & \multicolumn{3}{|c|}{ Glycemia $\geq 5.6 \mathrm{mmol} / \mathrm{L}$} & \multicolumn{3}{|c|}{ Insulin $\geq 20 \mu \mathrm{UI} / \mathrm{mL}$} \\
\hline & \multicolumn{2}{|c|}{ eGFR perc. } & \multirow[t]{2}{*}{$p$} & \multicolumn{2}{|c|}{ eGFR perc. } & \multirow{2}{*}{$p$} & \multicolumn{2}{|c|}{ eGFR perc. } & \multirow[t]{2}{*}{$p$} & \multicolumn{2}{|c|}{ eGFR perc. } & \multirow[t]{2}{*}{$p$} & \multicolumn{2}{|c|}{ eGFR perc. } & \multirow{2}{*}{$p$} & \multicolumn{2}{|c|}{ eGFR perc. } & \multirow{2}{*}{$p$} \\
\hline & $\leq 5$ th & $\geq 95$ th & & $\leq 5$ th & $\geq 95$ th & & $\leq 5$ th & $\geq 95$ th & & $\leq 5$ th & $\geq 95$ th & & $\leq 5$ th & $\geq 95$ th & & $\leq 5$ th & $\geq 95$ th & \\
\hline Sch-L & 1 & 4 & 0.363 & 9 & 8 & 0.797 & 0 & 2 & 0.495 & 3 & 1 & 0.363 & 2 & 2 & 1.000 & 0 & 4 & 0.118 \\
\hline Leger & 1 & 17 & $<0.001$ & 1 & 30 & $<0.001$ & 0 & 11 & 0.001 & 4 & 3 & 1.000 & 2 & 2 & 1.000 & 1 & 9 & 0.016 \\
\hline LM & 1 & 7 & 0.060 & 6 & 11 & 0.290 & 0 & 2 & 0.495 & 4 & 0 & 0.118 & 1 & 2 & 1.000 & 0 & 4 & 0.118 \\
\hline LM-LBM & 1 & 16 & $<0.001$ & 2 & 29 & $<0.001$ & 0 & 10 & 0.001 & 3 & 3 & 1.000 & 2 & 2 & 1.000 & 0 & 9 & 0.003 \\
\hline FAS-QH & 2 & 4 & 0.697 & 10 & 6 & 0.286 & 0 & 1 & 1.000 & 3 & 2 & 0.675 & 1 & 2 & 1.000 & 0 & 3 & 0.243 \\
\hline FAS-QA & 0 & 5 & 0.057 & 6 & 9 & 0.579 & 0 & 3 & 0.243 & 4 & 1 & 0.363 & 1 & 1 & 1.000 & 0 & 4 & 0.118 \\
\hline \multirow[t]{3}{*}{ Formula } & \multicolumn{3}{|c|}{$\mathrm{HDL}-\mathrm{C}<1.29 \mathrm{mmol} / \mathrm{L}$} & \multicolumn{3}{|c|}{$\mathrm{TAG} \geq 1.7 \mathrm{mmol} / \mathrm{L}$} & \multicolumn{3}{|c|}{ AIP $\geq 0.11$} & \multicolumn{3}{|c|}{$\mathrm{UA} \geq 340 \mu \mathrm{mol} / \mathrm{L}$} & \multicolumn{3}{|c|}{$\mathrm{ACR} \geq 3.5 \mathrm{mg} / \mathrm{mmol}$} & \multicolumn{3}{|c|}{$\mathrm{CRP}>3 \mathrm{mg} / \mathrm{L}$} \\
\hline & \multicolumn{2}{|c|}{ eGFR perc } & \multirow[t]{2}{*}{$p$} & \multicolumn{2}{|c|}{ eGFR perc } & \multirow[t]{2}{*}{$p$} & \multicolumn{2}{|c|}{ eGFR perc } & $p$ & eGF & perc & $p$ & eGF & perc & $p$ & eGF & R perc & $p$ \\
\hline & $\leq 5$ th & $\geq 95$ th & & $\leq 5$ th & $\geq 95$ th & & $\leq 5$ th & $\geq 95$ th & & $\leq 5$ th & $\geq 95$ th & & $\leq 5$ th & $\geq 95$ th & & $\leq 5$ th & $\geq 95$ th & \\
\hline Sch-L & 9 & 17 & 0.131 & 4 & 1 & 0.205 & 2 & 2 & 1.000 & 8 & 2 & 0.052 & 2 & 2 & 1.000 & 5 & 8 & 0.555 \\
\hline Leger & 5 & 22 & $<0.001$ & 2 & 3 & 1.000 & 1 & 5 & 0.205 & 7 & 5 & 0.761 & 4 & 0 & 0.118 & 5 & 14 & 0.041 \\
\hline LM & 9 & 18 & 0.073 & 3 & 1 & 0.618 & 2 & 1 & 1.000 & 9 & 3 & 0.123 & 2 & 2 & 1.000 & 4 & 7 & 0.526 \\
\hline LM-LBM & 5 & 20 & 0.001 & 2 & 3 & 1.000 & 1 & 5 & 0.205 & 8 & 5 & 0.555 & 3 & 0 & 0.243 & 4 & 13 & 0.032 \\
\hline FAS-QH & 7 & 15 & 0.094 & 2 & 1 & 0.614 & 0 & 2 & 0.495 & 4 & 2 & 0.433 & 2 & 2 & 1.000 & 2 & 6 & 0.271 \\
\hline FAS-QA & 8 & 17 & 0.066 & 3 & 1 & 0.618 & 2 & 1 & 1.000 & 10 & 2 & 0.028 & 2 & 1 & 1.000 & 4 & 7 & 0.526 \\
\hline
\end{tabular}

The prevalence is given as the number of females presenting the risk factor out of 53/group.

Formulae: Sch-L, Schwartz-Lyon; LM, Lund-Malmo; LM-LMB, Lund-Malmo with lean body mass; FAS-QH, Full-age Spectrum with Q-height; FAS-QA, Full-age Spectrum with Q-age.

WHtR, waist-to-height ratio; BMI, body mass index; ow/ob, overweight/obese; OITF, The International Obesity Taskforce; SBP, systolic blood pressure; DBP, diastolic blood pressure; HDL-C, high-density lipoprotein cholesterol; eGFR, estimated glomerular filtration rate; TAG, triacylglycerols; AIP, atherogenic index of plasma; ACR, UA, uric acid; urinary albumin-to-creatinine ratio; CRP, C-reactive protein, significant p is given in bold. 
in eGFR values returned by different formulae. Across eGFR percentiles, the trajectories returned by different formulae largely were not parallel, and the differences between eGFR values rose more steeply in males than females. We also revealed a substantial mismatch between formulae in assigning the subjects into the upper and lower tails of eGFR distributions. In females, two equations-Léger's and LM-LBM formulaeshowed a clinically acceptable match in assigning the subjects into both the lower and upper tails of eGFR distribution. However, adolescents of both sexes classified by these two equations as hyperfiltrating manifested a higher prevalence of obesity and obesity-associated comorbidities compared with their hypofiltrating peers. Adolescents assigned into the lower eGFR tails did not present an increased prevalence of comorbid conditions consistent with CKD except for a moderately higher prevalence of hyperuricemia in females with GFR $\leq 5$ th percentile according to the FAS-QA formula.

In line with former studies in healthy or T1D adolescents $(13,14,16)$ and in our study, different pediatric formulae returned diverse mean eGFR values. Five out of six equations indicated sex differences in mean eGFR that were inconsistent regarding whether mean eGFR was higher in males or females. The sex differences did not match completely the sex differences reported by Boettcher et al. (16) for a large group of children and adolescents with T1D. The difference between the highest and the lowest mean eGFR in our study was similar to that reported for 12 -17-year-old U.S. adolescents $\left(43 \mathrm{~mL} / \mathrm{min} / 1.73 \mathrm{~m}^{2}\right)$ (13) but was higher than the difference of about $16 \mathrm{~mL} / \mathrm{min} / 1.73 \mathrm{~m}^{2}$ in males and $20 \mathrm{~mL} / \mathrm{min} / 1.73 \mathrm{~m}^{2}$ in females observed in 1 - to $<18$-year-old T1D patients (16).

Differences among serum creatinine-based formulae derived to estimate GFR in the pediatric population stem from the differences in variables included in the equations (i.e., age, height, body weight, and sex), mathematical forms, and coefficients calculated to fit best the source data, e.g., the populations that were used to derive the equations. Among six formulae compared in our study, data on patients with kidney diseases served as sources in the construction of four of them. Schwartz-Lyon (18) and Léger's (19) formulae were derived from pediatric patients with $\mathrm{CKD}$, or CKD and kidney-transplanted patients, respectively. The LM and LM-LBM equations were developed from data on adults mostly with renal disease (21), and later they were evaluated in a pediatric cohort consisting mainly of subjects with suspected or confirmed CKD (31). It is suggested that, as in adults, also in children and adolescents equations developed in populations with decreased GFR underestimate GFR among those without kidney disease (13). However, in our cohort, Léger's formula consistently overestimated eGFR compared with values returned by FAS-QA and FAS-QH equations in both sexes, and in females, LM-LBM gave slightly higher eGFR means than both FAS formulae between the 1st to about the 25th percentile. It is assumed that variations in creatininemia in patients with CKD are more likely to reflect changes in GFR. Muscle mass, growth, or protein intake are more important determinants of serum creatinine levels in subjects without CKD. In our study, the LM equation gave the lowest eGFR among the six formulae throughout the whole range of creatinine values in both sexes and, thus, the highest prevalence $(>40 \%)$ of subjects with hypofiltration (eGFR $<75 \mathrm{~mL} / \mathrm{min} / 1.73 \mathrm{~m}^{2}$ ), followed by the Schwartz-Lyon formula. This is in line with data on 1 - to $<18$ year-old males with T1D although, in females, the SchwartzLyon formula gave lower means compared with the LM equation (16). As in the study of Boettcher et al. (16), the introduction of a lean body mass component into the LM equation returned higher mean eGFR compared with the LM equation. However, with GFR above $110 \mathrm{~mL} / \mathrm{min} / 1.73 \mathrm{~m}^{2}$, the overestimation of eGFR by the LM-LBM equation compared with LM and SchwartzLyon formulae gradually diminished (Figures 1A,B). Although both LM equations were derived from the same population, their trajectories across eGFR percentiles were not parallel. Léger's (19) formula returned the highest eGFR values throughout the whole serum creatinine range and showed the steepest rise across the percentiles. The difference between the 1st and 99th eGFR percentile reached a difference of $100 \mathrm{~mL} / \mathrm{min} / 1.73 \mathrm{~m}^{2}$ in males and $80 \mathrm{~mL} / \mathrm{min} / 1.73 \mathrm{~m}^{2}$ in females. With the other formulae derived from patients with $\mathrm{CKD}$, differences between the 1st and the 99th eGFR percentile varied between 50 and $60 \mathrm{~mL} / \mathrm{min} / 1.73 \mathrm{~m}^{2}$ in males and 40 and $50 \mathrm{~mL} / \mathrm{min} / 1.73 \mathrm{~m}^{2}$ in females. Nonetheless, for Léger's equation, the study in U.S. adolescents reported a similar difference in eGFR (about 120 $\mathrm{mL} / \mathrm{min} / 1.73 \mathrm{~m}^{2}$ ) across the percentiles (13) as observed by us. Of note, creatininemia ranged about $45-100 \mu \mathrm{mol} / \mathrm{L}$ in both studies.

The FAS-QA and FAS-QH formulae intended to provide equations for all ages without the discontinuity between pediatric and adult equations based on Belgian data on 0.1-20-yearold healthy subjects (15). The FAS-QA formula enables eGFR calculation in case the anthropometric data is not available. In line with findings in adolescents with T1D (16), in our females, both FAS equations returned similar mean eGFR although, in males, mean FAS-QH eGFR was slightly higher than that given by FAS-QA. In both sexes, the FAS-QA formula overestimated eGFR in comparison with the LM and SchwartzLyon formulae, but the rise in eGFR across the percentiles was similar. FAS-QH formula-derived eGFR rose sharply across the percentiles-particularly in males, in whom the difference across the percentiles corresponded to that observed for Léger's equation, and at the upper end of the distribution, two formulae returned almost identical eGFR values. This finding is surprising as it has been assumed that eGFR values returned by equations based on data from patients with renal disease differ from those derived from healthy subjects (5), and that the FAS$\mathrm{QH}$ equation outperforms the other height-dependent formulae in healthy adolescents (15). Likewise, the discrepancy between FAS-QA and FAS-QH formulae, particularly in our males, is surprising. It suggests that a single normalization constant derived from a population of healthy Belgian adolescents might not be representative of other European adolescents.

Theoretically, numerically different eGFR values returned by different formulae would not be confounding if reported along with age- and sex-specific reference ranges. Knowing which formula had been used and what the particular cutoffs are, the clinician or epidemiologist provided with a simple eGFR value would be able to judge whether an individual should be subjected to further diagnostic steps. However, this assumption would be 
plausible only if different formulae consistently classify the same subject as hypofiltrating or hyperfiltrating. This requirement is important for both general practice and epidemiological studies as subjects with low or high eGFR should be referred for further examination to confirm or reject kidney disease. Our data suggest that, in apparently healthy adolescents, a conventional cutoff limit for hypofiltration or hyperfiltration cannot be universally applied. The LM formula-derived eGFR yielded extremely high frequencies of eGFR $\leq 75 \mathrm{~mL} / \mathrm{min} / 1.73 \mathrm{~m}^{2}$ in both sexes, and the Schwartz-Lyon formula probably also overestimated the prevalence in our males. Applying the other formulae, the frequencies of abnormal eGFR were low and corresponded with those reported for the general population of adolescents in other studies $(13,23)$. On the other hand, Léger's and FAS-QH yielded a very high prevalence of $e G F R \geq 135 \mathrm{~mL} / \mathrm{min} / 1.73 \mathrm{~m}^{2}$ in both sexes, and Sch-L, LM, and LM-LBM hardly identified a single hyperfiltrating individual.

Similarly, a concordance of six formulae in assigning the same subject to the lowest or the highest $5 \%$ of eGFR distribution was poor. In both sexes, among 15 possible pairs between six formulae, the coefficient of determination $\geq 90 \%$ was revealed for three pairs (LM vs. Sch-L or FAS-QA and Léger vs. LMLBM). However, overall correlations seemed not to be sensitive enough to detect variations occurring at the extremities of the distribution. Although in females these three pairs of equations also showed acceptable matching $(\geq 80 \%)$ for assigning the probands into the lowest tail of eGFR distribution; in males, it was only LM vs. FAS-QA and, additionally, the Sch-L vs. LM-LBM pair. The differences in eGFR calculated by different formulae increase with higher $\operatorname{eGFR}(13,14)$. However, we show that, even the values at the 5th percentile are diverse: the height-independent formulae (LM and FAS-QA) showing an acceptable matching for classification of hypofiltration in both sexes, return at the 5th percentile values differing by $\geq 20 \mathrm{~mL} / \mathrm{min} / 1.73 \mathrm{~m}^{2}$. Regarding hyperfiltration, LM vs. Sch-L and Léger's formula vs. the LM-LMB showed $\geq 80 \%$ matching in adolescent males; in females, only the latter pair returned acceptable concordance. Our data do not support our hypothesis on the consistent classification of subjects to the lower or the upper tail of eGFR distribution by formulae derived from the same populations. As in apparently healthy adolescents, eGFR results indicating hypofiltration or hyperfiltration estimated by different creatinine-based formulae are equivocal. The interpretation of the results must be done with caution. This underscores the importance of clinical decision making, which includes multiple factors in addition to eGFR (32). Our data suggest that the question on the reliability of different formulae in the estimation of GFR (33) is not restricted to adults.

Except for the fact that both height-independent formulae assigned shorter individuals into the lower tail of eGFR distributions and that the FAS-QA equation indicated a moderately higher prevalence of hyperuricemia in females with eGFR $\leq 5$ th percentile compared with those within the upper $5 \%$, no group of participants with eGFR $\leq 5$ th percentile showed an increased prevalence of other conditions consistent with CKD. These findings are in line with those reported for U.S. adolescents in the lower ranges of eGFR (13). On the other hand, using Léger's and LM-LBM formulae, hyperfiltrating subjects of both sexes presented a higher prevalence of general and central obesity, dyslipidemia, fasting hyperinsulinemia, and CRP $>3 \mathrm{mg} / \mathrm{L}$ compared with their hypofiltrating peers. The U.S. study in nondiabetic adolescents also reported an association of glomerular hyperfiltration with hypertriacylglycerolemia and lower insulin sensitivity (34).

The strengths of our study are a reasonably large sample of White Caucasian adolescents for whom data were gathered within one school year, that morphometry was performed by trained staff according to the same protocol, and serum samples were analyzed in a central laboratory referencing creatinine assay to IDMS standards. We do not have data on renal or plasma creatinine clearance or the measured GFR; thus, we only could assess agreement between the formulae rather than which formula is most accurate. Observed associations might be biased by the potential participation of close relatives. We have no data on factors potentially influencing eGFR, such as birth weight, prematurity, history of former urinary tract infections, lifestyle and genetic factors, or family history of CKD. Our results are based on a single measurement, and we did not follow renal function status over time. There are limitations of generalizing our findings to populations with different epidemiological, anthropometric, or clinical characteristics.

In conclusion, our data show that relationships between eGFR values returned by pediatric formulae are largely lax, and the concordance of the equations in assigning apparently healthy adolescents as hypofiltrating or hyperfiltrating is generally poor. We did not follow renal function status over time, and to our knowledge, there is no data from other populations to compare potential discrepancies in the assignment of adolescents to tails of eGFR distributions. Thus, it remains questionable which eGFR formula should be used in adolescents to screen for abnormal renal function in general practice or epidemiological studies. This presents an opportunity for future studies in longitudinal cohorts.

\section{DATA AVAILABILITY STATEMENT}

The raw data supporting the conclusions of this article will be made available by the authors, without undue reservation.

\section{ETHICS STATEMENT}

The studies involving human participants were reviewed and approved by the Ethics Committee of Bratislava Self-Governing Region. Written informed consent to participate in this study was provided by the participants' legal guardian/next of kin.

\section{AUTHOR CONTRIBUTIONS}

KS and RG: drafting of the manuscript, statistical analysis, and had full access to all of the data in the study and take responsibility for the integrity of the data and the accuracy of the data analysis. KŠ and ĹP: study concept and design. KŠ, RG, and LT: acquisition of data. KS: study supervision. All authors: critical 
revision of the manuscript for important intellectual content, analysis, or interpretation of data.

\section{FUNDING}

This work was supported by grants from The Slovak Research and Development Agency No. APVV-0447-12, and APVV-180287; The Scientific Grant Agency of the Ministry of Education, Science, Research and Sport of the Slovak Republic and the Slovak Academy of Sciences No. 1/0637/13; and the Bratislava Self-governing Region.

\section{REFERENCES}

1. Vivante A, Golan E, Tzur D, Leiba A, Tirosh A, Skorecki K, et al. Body mass index in 1.2 million adolescents and risk for end-stage renal disease. Arch Intern Med. (2012) 172:1644-50. doi: 10.1001/2013.jamainternmed.85

2. Ruiz LD, Zuelch ML, Dimitratos SM, Scherr RE. Adolescent obesity: diet quality, psychosocial health, and cardiometabolic risk factors. Nutrients. (2019) 12:43. doi: 10.3390/nu12010043

3. Leiba A, Fishman B, Twig G, Gilad D, Derazne E, Shamiss A, et al. Association of adolescent hypertension with future end-stage renal disease. JAMA Intern Med. (2019) 179:517-23. doi: 10.1001/jamainternmed.2018.7632

4. Filler G, Yasin A, Medeiros M. Methods of assessing renal function. Pediatr Nephrol. (2014) 29:183-92. doi: 10.1007/s00467-013-2426-7

5. Pottel H. Measuring and estimating glomerular filtration rate in children. Pediatr Nephrol. (2017) 32:249-63. doi: 10.1007/s00467-016-3373-x

6. Roos JF, Doust J, Tett SE, Kirkpatrick CM. Diagnostic accuracy of cystatin $\mathrm{C}$ compared to serum creatinine for the estimation of renal dysfunction in adults and children-a meta-analysis. Clin Biochem. (2007) 40:38391. doi: 10.1016/j.clinbiochem.2006.10.026

7. Ferguson TW, Komenda P, Tangri N. Cystatin C as a biomarker for estimating glomerular filtration rate. Curr Opin Nephrol Hypertens. (2015) 24:295300. doi: 10.1097/MNH.0000000000000115

8. Levey AS, Bosch JP, Lewis JB, Greene T, Rogers N, Roth D, et al. A more accurate method to estimate glomerular filtration rate from serum creatinine: a new prediction equation. Modification of Diet in Renal Disease Study Group. Ann Intern Med. (1999) 130:46170. doi: 10.7326/0003-4819-130-6-199903160-00002

9. Levey AS, Stevens LA, Schmid CH, Zhang YL, Castro AF 3rd, Feldman HI, et al. A new equation to estimate glomerular filtration rate. Ann Intern Med. (2009) 150:604-12. doi: 10.7326/0003-4819-150-9-200905050-00006

10. Chehade H, Girardin E, Iglesias K, Ramseyer P, Frey P, Bardy D, et al. Assessment of adult formulas for glomerular filtration rate estimation in children. Pediatr Nephrol. (2013) 28:105-14. doi: 10.1007/s00467-012-2298-2

11. Azzi A, Cachat F, Faouzi M, Mosig D, Ramseyer P, Girardin E, et al. Is there an age cutoff to apply adult formulas for GFR estimation in children? J Nephrol. (2015) 28:59-66. doi: 10.1007/s40620-014-0148-y

12. Selistre L, Rabilloud M, Cochat P, De Souza V, Iwaz J, Lemoine $S$, et al. Comparison of the Schwartz and CKD-EPI equations for estimating glomerular filtration rate in children, adolescents, and adults: a retrospective cross-sectional study. PLoS Med. (2016) 13:e1001979. doi: 10.1371/journal.pmed.1001979

13. Fadrowski JJ, Neu AM, Schwartz GJ, Furth SL. Pediatric GFR estimating equations applied to adolescents in the general population. Clin J Am Soc Nephrol. (2011) 6:1427-35. doi: 10.2215/CJN.06460710

14. Zachwieja K, Korohoda P, Kwinta-Rybicka J, Miklaszewska M, Moczulska A, Bugajska J, et al. Which equations should and which should not be employed in calculating eGFR in children? Adv Med Sci. (2015) 60:3140. doi: 10.1016/j.advms.2014.08.007

15. Hoste L, Dubourg L, Selistre L, De Souza VC, Ranchin B, Hadj-Aïssa A, et al. A new equation to estimate the glomerular filtration rate in children, adolescents and young adults. Nephrol Dial Transplant. (2014) 29:108291. doi: $10.1093 / \mathrm{ndt} / \mathrm{gft} 277$

16. Boettcher C, Utsch B, Galler A, Grasemann C, Borkenstein M, Denzer C, et al. Estimated glomerular filtration rates calculated by new and old equations in

\section{ACKNOWLEDGMENTS}

The authors thank all those who put their effort into the accomplishment of the Respect for Health study.

\section{SUPPLEMENTARY MATERIAL}

The Supplementary Material for this article can be found online at: https://www.frontiersin.org/articles/10.3389/fped. 2021.719997/full\#supplementary-material

children and adolescents with type 1 diabetes-what to do with the results? Front Endocrinol (Lausanne). (2020) 11:52. doi: 10.3389/fendo.2020.00052

17. Gurecka R, Koborova I, Sebek J, Sebekova K. Presence of cardiometabolic risk factors is not associated with microalbuminuria in 14-to-20-years old slovak adolescents: a cross-sectional, population study. PLoS One. (2015) 10:e0129311. doi: 10.1371/journal.pone.0129311

18. De Souza VC, Rabilloud M, Cochat P, Selistre L, Hadj-Aissa A, Kassai B, et al. Schwartz formula: is one k-coefficient adequate for all children? PLoS One. (2012) 7:e53439. doi: 10.1371/journal.pone.0053439

19. Léger F, Bouissou F, Coulais Y, Tafani M, Chatelut E. Estimation of glomerular filtration rate in children. Pediatr Nephrol. (2002) 17:9037. doi: 10.1007/s00467-002-0964-5

20. Björk J, Grubb A, Sterner G, Nyman U. Revised equations for estimating glomerular filtration rate based on the Lund-Malmö Study cohort. Scand J Clin Lab Invest. (2011) 71:232-9. doi: 10.3109/00365513.2011.557086

21. Björk J, Bäck SE, Sterner G, Carlson J, Lindstrom V, Bakoush O, et al. Prediction of relative glomerular filtration rate in adults: new improved equations based on Swedish Caucasians and standardized plasma-creatinine assays. Scand J Clin Lab Invest. (2007) 67:678-95. doi: 10.1080/00365510701326891

22. Cachat F, Combescure C, Cauderay M, Girardin E, Chehade H. A systematic review of glomerular hyperfiltration assessment and definition in the medical literature. Clin J Am Soc Nephrol. (2015) 10:382-9. doi: 10.2215/CJN.03080314

23. Pottel H, Hoste L, Delanaye P. Abnormal glomerular filtration rate in children, adolescents and young adults starts below $75 \mathrm{~mL} / \mathrm{min} / 1.73 \mathrm{~m}^{2}$. Pediatr Nephrol. (2015) 30:821-8. doi: 10.1007/s00467-014-3002-5

24. Cole TJ, Bellizzi MC, Flegal KM, Dietz WH. Establishing a standard definition for child overweight and obesity worldwide: international survey. BMJ. (2000) 320:1240-3. doi: 10.1136/bmj.320.7244.1240

25. Ashwell M, Gibson S. A proposal for a primary screening tool: 'Keep your waist circumference to less than half your height'. BMC Med. (2014) 12:207. doi: 10.1186/s12916-014-0207-1

26. Dobiasova M, Frohlich J. The plasma parameter log (TG/HDL-C) as an atherogenic index: correlation with lipoprotein particle size and esterification rate in apoB-lipoprotein-depleted plasma (FER(HDL)). Clin Biochem. (2001) 34:583-8. doi: 10.1016/S0009-9120(01)00263-6

27. Sebekova K, Stefikova K, Polakovicova D, Spustova V, Dzurik R. Does magnesium dysbalance participate in the development of insulin resistance in early stages of renal disease? Physiol Res. (2002) 51:605-12.

28. Ridker PM. Clinical application of C-reactive protein for cardiovascular disease detection and prevention. Circulation. (2003) 107:363-9. doi: 10.1161/01.CIR.0000053730.47739.3C

29. Johnson DW, Jones GR, Mathew TH, Ludlow MJ, Chadban SJ, Usherwood T, et al. Chronic kidney disease and measurement of albuminuria or proteinuria: a position statement. Med J Aust. (2012) 197:224-5. doi: 10.5694/mjal1. 11468

30. Experts. Physical Development of Children and Adolescents. Results of the VI. Nationwide Survey in 2011. Bratislava: Public Health Authority of the Slovak Republic (2012). Available online at: https://www.uvzsr.sk/docs/info/ hdm/Antropometria.pdf (accessed January 13, 2020) (in Slovak).

31. Nyman U, Björk J, Lindström V, Grubb A. The Lund-Malmö creatininebased glomerular filtration rate prediction equation for adults also performs well in children. Scand J Clin Lab Invest. (2008) 68:56876. doi: 10.1080/00365510801915163 
32. Levey AS, Coresh J, Tighiouart H, Greene T, Inker LA. Strengths and limitations of estimated and measured GFR. Nat Rev Nephrol. (2019) 15:784. doi: 10.1038/s41581-019-0213-9

33. Porrini E, Ruggenenti P, Luis-Lima S, Carrara F, Jiménez A, De Vries APJ, et al. Estimated GFR: time for a critical appraisal. Nat Rev Nephrol. (2019) 15:177-90. doi: 10.1038/s41581-018-0080-9

34. Lee AM, Charlton JR, Carmody JB, Gurka MJ, Deboer MD. Metabolic risk factors in nondiabetic adolescents with glomerular hyperfiltration. Nephrol Dial Transplant. (2017) 32:1517-24. doi: 10.1093/ndt/ gfw231

Conflict of Interest: The authors declare that the research was conducted in the absence of any commercial or financial relationships that could be construed as a potential conflict of interest.
Publisher's Note: All claims expressed in this article are solely those of the authors and do not necessarily represent those of their affiliated organizations, or those of the publisher, the editors and the reviewers. Any product that may be evaluated in this article, or claim that may be made by its manufacturer, is not guaranteed or endorsed by the publisher.

Copyright (C) 2021 Šebeková, Gurecká, Tóthová and Podracká. This is an open-access article distributed under the terms of the Creative Commons Attribution License (CC $B Y)$. The use, distribution or reproduction in other forums is permitted, provided the original author(s) and the copyright owner(s) are credited and that the original publication in this journal is cited, in accordance with accepted academic practice. No use, distribution or reproduction is permitted which does not comply with these terms. 